\section{E-151 VOLUMETRIC ANALYSIS OF ENDOSCOPIC EVACUATION OF INTRACEREBRAL HEMORRHAGE}

${ }^{1} \mathrm{G}$ Barros*, ${ }^{1} \mathrm{R}$ Kellogg, ${ }^{1} \mathrm{~J}$ Keen, ${ }^{1} \mathrm{C}$ Kelly, ${ }^{2} \mathrm{~A}$ Lele, ${ }^{1} \mathrm{~L}$ Kim, ${ }^{1} \mathrm{M}$ Levitt. ${ }^{1}$ Neurosurgery, University of Washington, Seattle, WA; ${ }^{2}$ Anesthesiology/Critical Care, University of Washington, Seattle, WA

\subsection{6/neurintsurg-2020-SNIS.183}

Introduction Spontaneous intracerebral hemorrhage (ICH) is a neurologically-devastating form of stroke. Minimally-invasive evacuation is increasingly investigated as a primary treatment. Secondary analyses of the MISTIE III (Minimally Invasive Surgery Plus Alteplase for Intracerebral Hemorrhage Evacuation) trial, which used stereotactic catheter drainage, demonstrated promising results among functional outcomes within the surgical arm. A sustained functional outcome advantage was found among patients with less than $15 \mathrm{~mL}$ residual clot volume or greater than $70 \%$ volume reduction. There is limited research on the effect of post-surgical ICH volumes on functional outcomes for patients specifically undergoing endoscopic evacuations.

Objective Our primary objective is to study how the residual volume and percentage volume reduction of $\mathrm{ICH}$ after endoscopic evacuation affects the functional outcome of surgical patients. Methods We conducted a retrospective review of all endoscopic ICH evacuations performed between October 2016 and February 2020 by our institution's cerebrovascular neurosurgery service. Demographics, comorbidities, ICH radiographic characteristics, cardiac status, intra-operative

\begin{tabular}{|c|c|}
\hline Age in years mean (SD) & $55.5(11.5)$ \\
\hline Sex Male $n(\%)$ & $23(74.2 \%)$ \\
\hline BMI mean (SD) & $31.1(7.4 \%)$ \\
\hline Admission GCS median [range] & $9[4-15]$ \\
\hline Intracerebral hemorrhage score median (range) & $2[0-4]$ \\
\hline \multicolumn{2}{|l|}{ Hemodynamics } \\
\hline Admission $\mathrm{SBP}$ mmHg mean (SD) & $157(34)$ \\
\hline Admission DBP mmHg mean (SD) & $102(29)$ \\
\hline Admission MAP mmHg mean (SD) & $118(29)$ \\
\hline Admission heart rate beats per minute mean (SD) & $76(15)$ \\
\hline \multicolumn{2}{|l|}{ Electrocardiographic findings } \\
\hline Normal sinus rhythm & $31(100 \%)$ \\
\hline Prolonged corrected QT interval & $18(58 \%)$ \\
\hline ST.T changes & $15(48.4 \%)$ \\
\hline Left ventricular hypertrophy & $7(22.6 \%)$ \\
\hline Atrial enlorgement & $5(16.2 \%)$ \\
\hline Atris-ventricular nodal block & $2(6.4 \%)$ \\
\hline Elevated troponin $(n=20)$ & $12(60 \%)$ \\
\hline Chest $x$-ray with pulmonary edema & $8(25.8 \%)$ \\
\hline \multicolumn{2}{|l|}{ Intracerebral hemorrhage characteristics } \\
\hline ICH side right & $10(33.2 \%)$ \\
\hline ICH location (deep) & $24(77.4 \%)$ \\
\hline Presence of intraventricular hemorrhage & $13(42 \%)$ \\
\hline Placement of external ventricular drain & $5(16.1 \%)$ \\
\hline Midline shift mm mean (SD) & $5.5(2.5)$ \\
\hline Pre-Operative ICH volume cu.ml mean (SO) & $54.3(24.4)$ \\
\hline Post-Operative ICH Volume $\mathrm{Cu} / \mathrm{ml}$ mean (SO) & $15.3(15.2)$ \\
\hline Percentaze Reduction mean (SO) & $72.8(24.4)$ \\
\hline Mechanical Ventilation Status & $14(48.4 \%)$ \\
\hline Intensive care unit length of stay in days mean (SD) & $8.3(9)$ \\
\hline Hospital length of stay in days mean (SD) & $49(95)$ \\
\hline Tracheostomy & $1(3 \%)$ \\
\hline Percutaneous gastrostomy feeding tube & $6(19.4 \%)$ \\
\hline \multicolumn{2}{|l|}{ Discharge disposition } \\
\hline In-hospital mortality & $2(6.5 \%)$ \\
\hline Home & $3(9.7 \%)$ \\
\hline Rehabilitation & $13(42 \%)$ \\
\hline Skilled nursing facility & $12(38.7 \%)$ \\
\hline \multicolumn{2}{|l|}{ Modified Rankin Score median[range] } \\
\hline At discharge $n=30$ & $5[3 \cdot 6]$ \\
\hline At 30 days $n=19$ & $4[3-5]$ \\
\hline At 90 months $n=18$ & $4[1-5]$ \\
\hline At 12 months $n=6$ & $4[1-4]$ \\
\hline
\end{tabular}

Abstract E-151 Table 1 Characteristics of patients $(n=31)$ undergoing minimally invasive evacuation of intracerebral hematoma vital signs, medications, post-operative clinical course, and functional outcomes measured as mRS (modified Rankin Scale) were assessed The mRS was assessed at discharge, 30 days, and $>90$ days. Pre-operative and immediate post-operative CT scans were evaluated. ICH volumes before and after evacuation were manually calculated using the $\mathrm{ABC} / 2$ method.

Results A total of 32 endoscopic evacuations among 31 patients were performed. The mean post-operative ICH volume was $15.3 \mathrm{~mL}$ (15.2), while the mean percentage volume reduction was $72.8 \%$ (24.4). There was a trend of lower post-operative ICH volumes correlating with lower mRS at discharge. Final data and analyses will be presented.

Conclusion Lower post-operative residual clot volumes correlate with improved functional outcome at discharge in patients undergoing minimally-invasive endoscopic evacuation of $\mathrm{ICH}$.

Disclosures G. Barros: None. R. Kellogg: None. J. Keen: None. C. Kelly: None. A. Lele: 1; C; LifeCenter Northwest, Aqueduct Critical Care. L. Kim: 2; C; Microvention, Inc. 4; C; Spi Surgical, Inc. M. Levitt: 1; C; Stryker, Medtronic, Philips Volcano. 2; C; Metis Innovative. 4; C; Synchron, Eloupes, Cerebrotech.

\section{E-152 SUBARACHNOID HEMORRHAGE QUANTITATIVE VOLUME ANALYSIS: BLOOD VOLUME PREDICTS CEREBRAL VASOSPASM, DELAYED CEREBRAL INFARCTION AND CLINICAL OUTCOME}

B Daou*, S Khalsa, S Anand, C Williamson, K Rajajee, K Sheehan, A Pandey. Neurosurgery, University of Michigan, Ann Arbor, MI

10.1136/neurintsurg-2020-SNIS.184

Introduction Conventional grading scales in aneurysmal subarachnoid hemorrhage (aSAH) do not take into account the total blood volume and have been criticized for being open to variability in interpretation. We aimed to evaluate an automatic tool for quantification of the hemorrhage volume in aSAH.

Methods Blood volumes were retrospectively analyzed and added to a comprehensive prospectively maintained institutional SAH database. Patients with non-aSAH were excluded. We designed an automatic quantitative blood volume analysis tool that utilizes a segmentation system that differentiates seed points corresponding to the different intracranial compartments. Through logistic regression analysis, we evaluated the association of blood volume with clinical cerebral vasospasm, delayed cerebral infarction (DCI) and 3-months clinical outcome. The diagnostic accuracy (AUC) of the quantitative blood volume analysis was compared to the modified Fisher score, the Hunt and Hess sore, the Hijdra score, and the World Federation of Neurosurgical Societies (WFNS) score.

Results 288 aSAH patients with a mean total blood volume of $74.9 \mathrm{ml}( \pm 39.7 \mathrm{ml}$ ) made up the study population. 73 patients developed clinical vasospasm (25.3\%) and 52 patients developed DCI (18.06\%). In univariate and multivariate analysis, total blood volume was significantly predictive of clinical vasospasm, DCI and clinical outcome. Blood volume had the highest AUC for clinical vasospasm as well as DCI when compared to conventional SAH scales. The modified Fisher score was not significantly predictive of either outcome. 
Conclusion Quantitative analysis of blood volume in aSAH provides a tool for prediction of cerebral vasospasm, DCI and clinical outcome that may have a higher performance and improved accuracy when compared to grading systems used in current practice.

Disclosures B. Daou: None. S. Khalsa: None. S. Anand: None. C. Williamson: None. K. Rajajee: None. K. Sheehan: None. A. Pandey: None.

\section{E-153 THE VALUE OF REPEATED DIGITAL SUBTRACTION ANGIOGRAPHY IN PATIENTS WITH INITIAL ANGIOGRAM-NEGATIVE PERIMESENCEPHALIC SUBARACHNOID HEMORRHAGE}

C Wipplinger*, C Preuss-Hernandez, M Fehn, J Klingenschmid, W Ho, A Goerke, C Thomé, 0 Petr. Department of Neurosurgery, Medical University of Innsbruck, Innsbruck, Austria

10.1136/neurintsurg-2020-SNIS. 185

Objective In approximately $15 \%$ of patients admitted with subarachnoid hemorrhage $(\mathrm{SAH})$ with a perimesencephalic blood distribution, digital subtraction angiography (DSA) fails to reveal a source of hemorrhage. These perimesencephalic non-aneurysmal hemorrhages usually have a benign clinical course and a significantly better prognosis than aneurysmal SAH. Recent studies hypothesized that repeated angiograms in this subset of patients does not add any valuable information. In the present study, we aimed to evaluate the value of repeated DSAs and complications in patients with perimesencephalic subarachnoid hemorrhage.

Methods We retrospectively reviewed the medical records of 73 patients with perimesencephalic subarachnoid hemorrhage and a negative initial angiogram admitted to our institution between 1998 and 2018. Findings of initial CT scans, DSAs as well as subsequent imaging including CT angiograms (CTA) and MR angiograms (MRA) were analyzed for significant findings as well as procedure-related complications.

Results All patients initially received a cranial CT scan followed by at least one DSA. In $41(56 \%)$ patients, DSA was repeated at least once and in $43(59 \%)$ patients a CTA was performed prior to the initial DSA. One patient (1.4\%) showed clinical deterioration and a recurrent hemorrhage on a follow-up CT scan. The subsequent DSA revealed a basilar artery dissection that was not identified on the initial DSA. Five $(6.8 \%)$ patients showed embolic strokes post DSA, requiring a decompressive suboccipital craniectomy in one (1.4\%) patient.

Conclusion In the majority of our patient population, repeated DSA did not add any clinically relevant information. However, if clinical deterioration with recurrent hemorrhage becomes apparent a repeated DSA may be indicated. Additionally, our findings show that DSA is an invasive procedure with potentially severe complications. Careful consideration is therefore necessary before indicating repeated DSAs.

Disclosures C. Wipplinger: None. C. Preuss-Hernandez: None. M. Fehn: None. J. Klingenschmid: None. W. Ho: None. A. Goerke: None. C. Thomé: None. O. Petr: None.

\section{E-154 INTERNAL CAROTID ARTERY INJURY DURING ENDOSCOPIC TRANSNASAL TRANSSPHENOIDAL SURGERY: A RETROSPECTIVE REVIEW}

G Wong*. Neurosurgery, The Chinese University of Hong Kong, Hong Kong, Hong kong

\subsection{6/neurintsurg-2020-SNIS. 186}

Introduction Internal carotid artery injury is a rare and severe intraoperative catastrophy. Endovascular treatment is the key to secure hemostasis. We aim to review our institutional results and learn from the experience.

Method We retrospectively reviewed all the patients undergoing neuro-endovascular intervention for the diagnosis of internal carotid artery injury during endoscopic transnasal transsphenoidal surgery between 2015 and 2019 in our institution.

Results We identified 4 patients with torrential intraoperative hemorrhage during endoscopic transnasal transphenoidal surgery requiring urgent catheter angiography. Three had diffuse subarachnoid hemorrhage and one had sole sellar hemorrhage. Diagnoses were pituitary marcoadenoma in 3 patients and mycosis in 1 patient. One aneurysm was embolized with coils and 2 patients were treated with main trunk occlusion together with the pseudoaneurysms. These 3 patients achieved favorable functional outcome upon discharge or after 